\title{
Lobar and segmental liver atrophy associated with hilar cholangiocarcinoma and the impact of hilar biliary anatomical variants: a pictorial essay
}

\author{
Brendon R. Friesen • Robert N. Gibson • Tony Speer • \\ Janette M. Vincent • Damien Stella $•$ Neil A. Collier
}

Received: 1 December 2010 /Revised: 19 February 2011 / Accepted: 2 May 2011 / Published online: 26 May 2011

(C) European Society of Radiology 2011

\begin{abstract}
The radiological features of lobar and segmental liver atrophy and compensatory hypertrophy associated with biliary obstruction are important to recognise for diagnostic and therapeutic reasons. Atrophied lobes/segments reduce in volume and usually contain crowded dilated bile ducts extending close to the liver surface. There is often a "step" in the liver contour between the atrophied and non-atrophied parts. Hypertrophied right lobe or segments enlarge and show a prominently convex or "bulbous" visceral surface. The atrophied liver parenchyma may show lower attenuation on pre-contrast computed tomography (CT) and CT intravenous cholangiography (CT-IVC) and lower signal intensity on T1weighted magnetic resonance imaging (MRI). Hilar biliary anatomical variants can have an impact on the patterns of lobar/segmental atrophy, as the cause of obstruction (e.g. cholangiocarcinoma) often commences in one branch, leading to atrophy in that drainage region before progressing to complete biliary obstruction and jaundice. Such variants are common and can result in unusual but explainable patterns of
\end{abstract}

B. R. Friesen · R. N. Gibson · J. M. Vincent • D. Stella Department of Radiology, Royal Melbourne Hospital, University of Melbourne,

Melbourne, Victoria 3050, Australia

T. Speer

Department of Gastroenterology, Royal Melbourne Hospital, Melbourne, Victoria 3050, Australia

\section{N. A. Collier}

Department of Surgery, Royal Melbourne Hospital,

Melbourne, Victoria 3050, Australia

\section{B. R. Friesen $(\triangle)$}

Department of Diagnostic Imaging, Monash Medical Centre, 246 Clayton Road,

Clayton, Victoria 3168, Australia

e-mail: Brendon.Friesen@southernhealth.org.au atrophy and hypertrophy. Examples of changes seen with and without hilar variants are presented that illustrate the radiological features of atrophy/hypertrophy.

Keywords Atrophy $\cdot$ Cholestasis · Bile duct diseases . Liver. Hypertrophy

\section{Introduction}

Lobar atrophy is well recognised in association with both benign and malignant biliary obstruction, the most common aetiologies being post-cholecystectomy stricture and cholangiocarcinoma respectively [1-3]. Benign strictures result in atrophy as a result of lobar/segmental biliary obstruction, whereas cholangiocarcinoma can result in atrophy because of biliary obstruction and/or portal vein branch compromise [3-5]. In either situation the contralateral lobe often hypertrophies $[3,6]$.

If cholangiocarcinoma arises in a right or left hepatic duct, the resulting obstruction, with or without portal vein branch compromise, may lead to asymptomatic atrophy of that lobe, with jaundice not occurring until the tumour extends centrally to involve the confluence of the right and left hepatic ducts [3-5].

It is important to recognise the presence of lobar and segmental liver atrophy and hypertrophy, as it influences the surgical and interventional management of biliary obstruction $[3,7]$.

This pictorial essay describes the radiological findings of lobar and segmental atrophy associated with hilar cholangiocarcinoma. The impact of hilar biliary anatomical variants, which are relatively common, is highlighted as these have not been described previously and can result in unusual findings. 


\section{Lobar atrophy}

Reduction in volume and ductal crowding

On cross-sectional imaging, lobar atrophy is usually defined as a reduction in the size of that lobe by at least $50 \%$ [8], or the presence of obvious ductal crowding on sectional imaging or cholangiography $[1-3,8-10]$ (Figs. 1, 2 and 3 ).

As the left lobe is normally smaller than the right, being $40-70 \%$ of the right lobe volume [11-15], it is important to factor this into assessment of size difference between the two lobes.

There is a reduction in the volume of hepatic parenchyma between the dilated bile ducts, so the actual parenchymal reduction is frequently greater than the overall lobar volume reduction. The dilated ducts consequently become more crowded and they appear to extend more peripherally towards the liver capsule, somewhat analogous to bronchial dilatation in bronchiectasis (Fig. 1).

With right lobe atrophy, the atrophic lobe tends to "rotate" more superiorly and posteriorly (Fig. 1). In left lobe atrophy, the lobe is smaller but otherwise not appreciably displaced (Fig. 3).

\section{Contour "step"}

There is often a change in hepatic contour, or a "step", between the atrophied and non-atrophied parts (Figs. 3 and 8). This "step" sign, when present, allows more confident
Fig. 1 a-e A patient with right lobe atrophy associated with cholangiocarcinoma. a-c Contrast-enhanced CT; d, e T2-weighted magnetic resonance imaging (MRI)/ magnetic resonance

cholangiopancreatogram (MRCP). a The right lobe is atrophied, and lies posteriorly with markedly dilated ducts. b The caudate lobe is probably hypertrophied (black arrow), and there is definite hypertrophy of segment IV (white arrow) and the left lateral segments (curved arrow). c The hypertrophied left lobe (segment III) extends inferiorly (white arrow). d, e Within the atrophied lobe, the ducts are markedly dilated and crowded (white arrow). e The ducts of the hypertrophied lobe are mildly dilated and spread out (arrowhead)
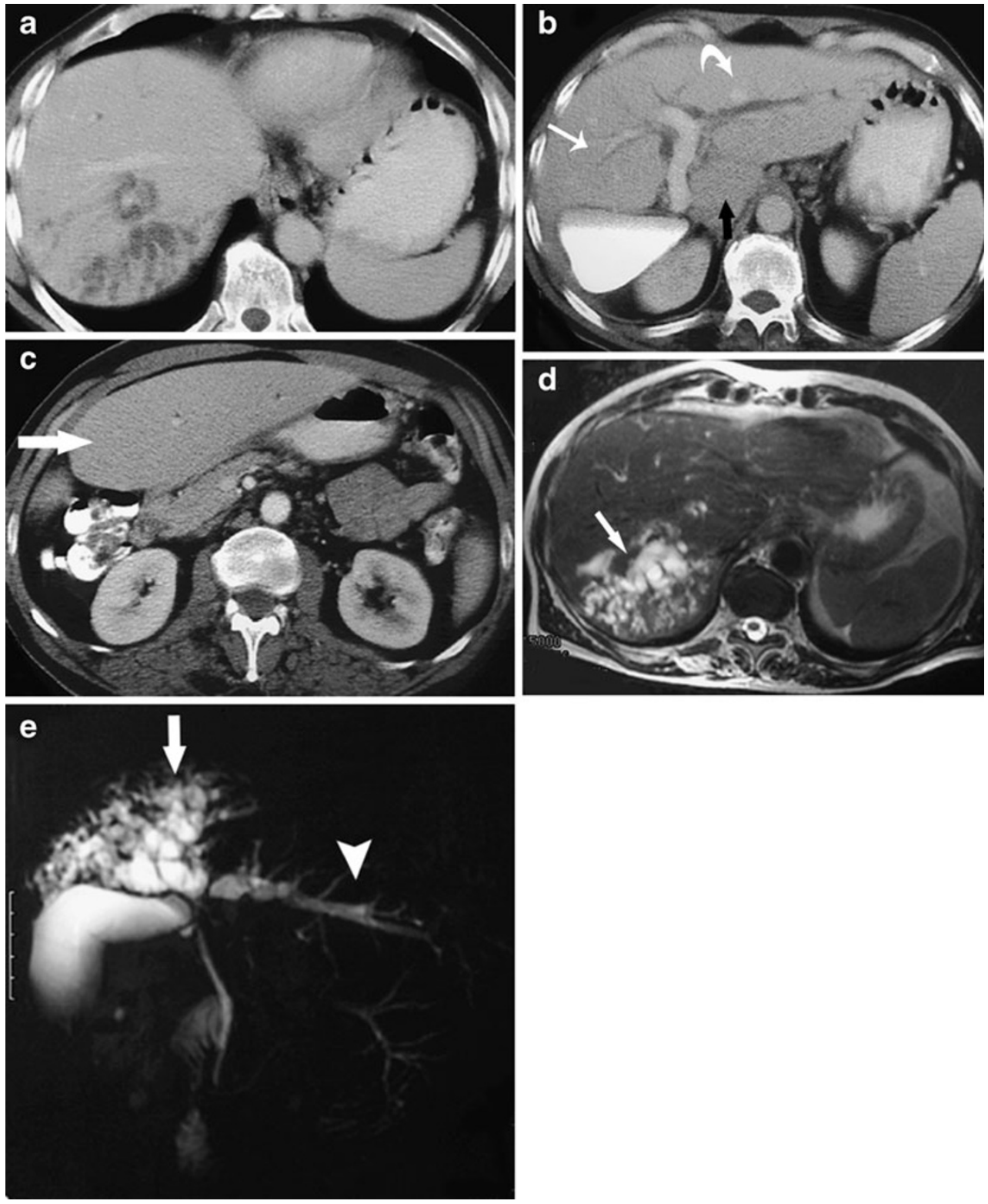
Fig. 2 a-c A patient with right lobar atrophy and left lobar hypertrophy associated with cholangiocarcinoma. a The right lobe is atrophic with markedly dilated ducts (arrow) on computed tomography (CT). The left lobe, and probably the caudate lobe (arrowhead), are hypertrophied. A stent is in place (curved arrow). b The atrophied lobe (arrow) shows lower attenuation relative to the non-atrophied lobe on CT-IVC. c CT-IVC surface-rendered reformat. The ducts draining segments II and III in the hypertrophied lobe are spread out (arrow), relative to the ducts of the atrophied lobe
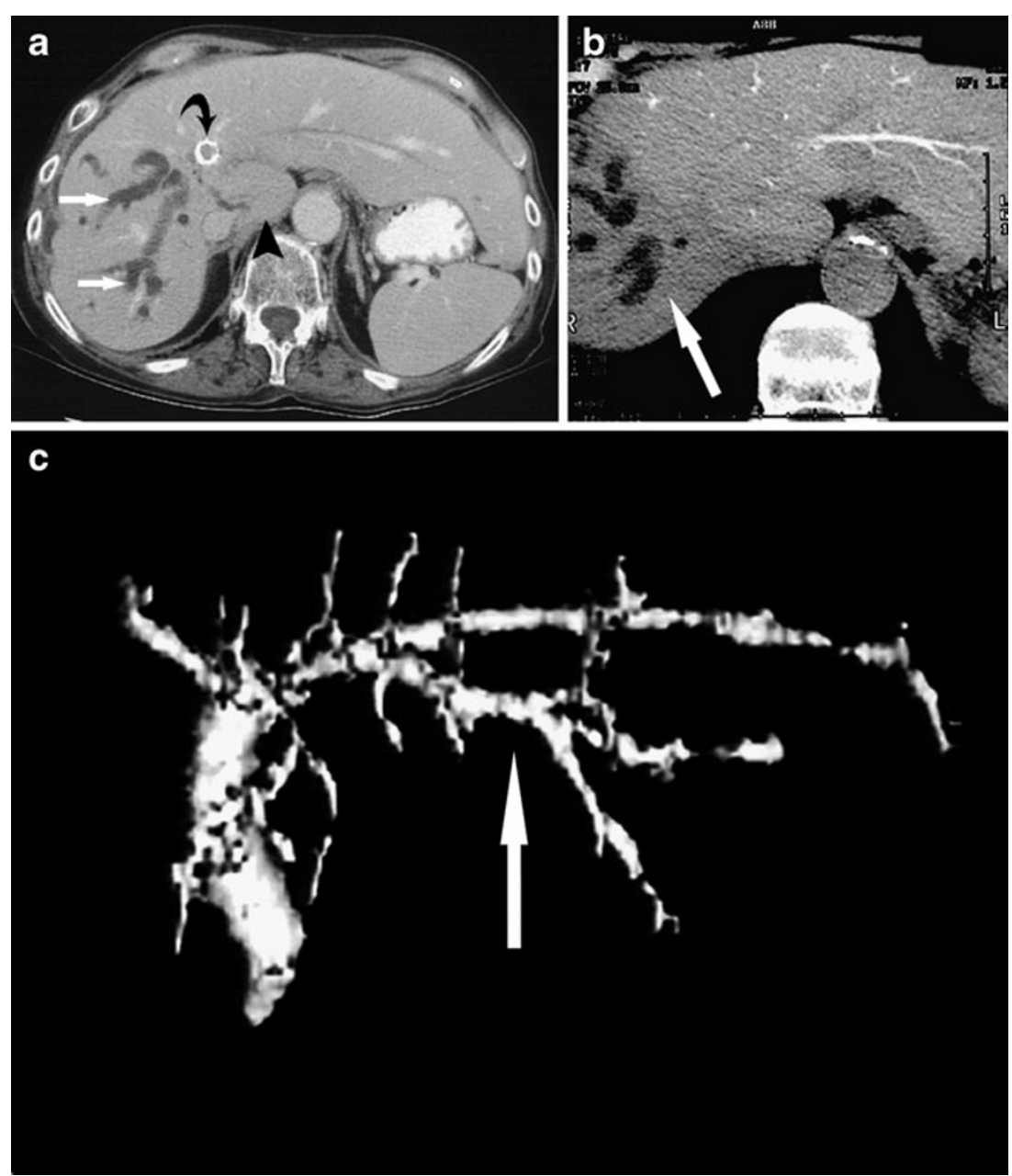

Fig. 3 a-c A patient with left lobe atrophy and right lobe hypertrophy associated with cholangiocarcinoma. a, b CT demonstrates a "step" in the contour between the atrophied and non-atrophied lobes (curved arrow). The ducts within the atrophied left lobe appear crowded and markedly dilated (arrowhead). The caudate lobe is clearly hypertrophied (arrow). c The hypertrophied right lobe has a "bulbous" visceral contour inferiorly (arrows)
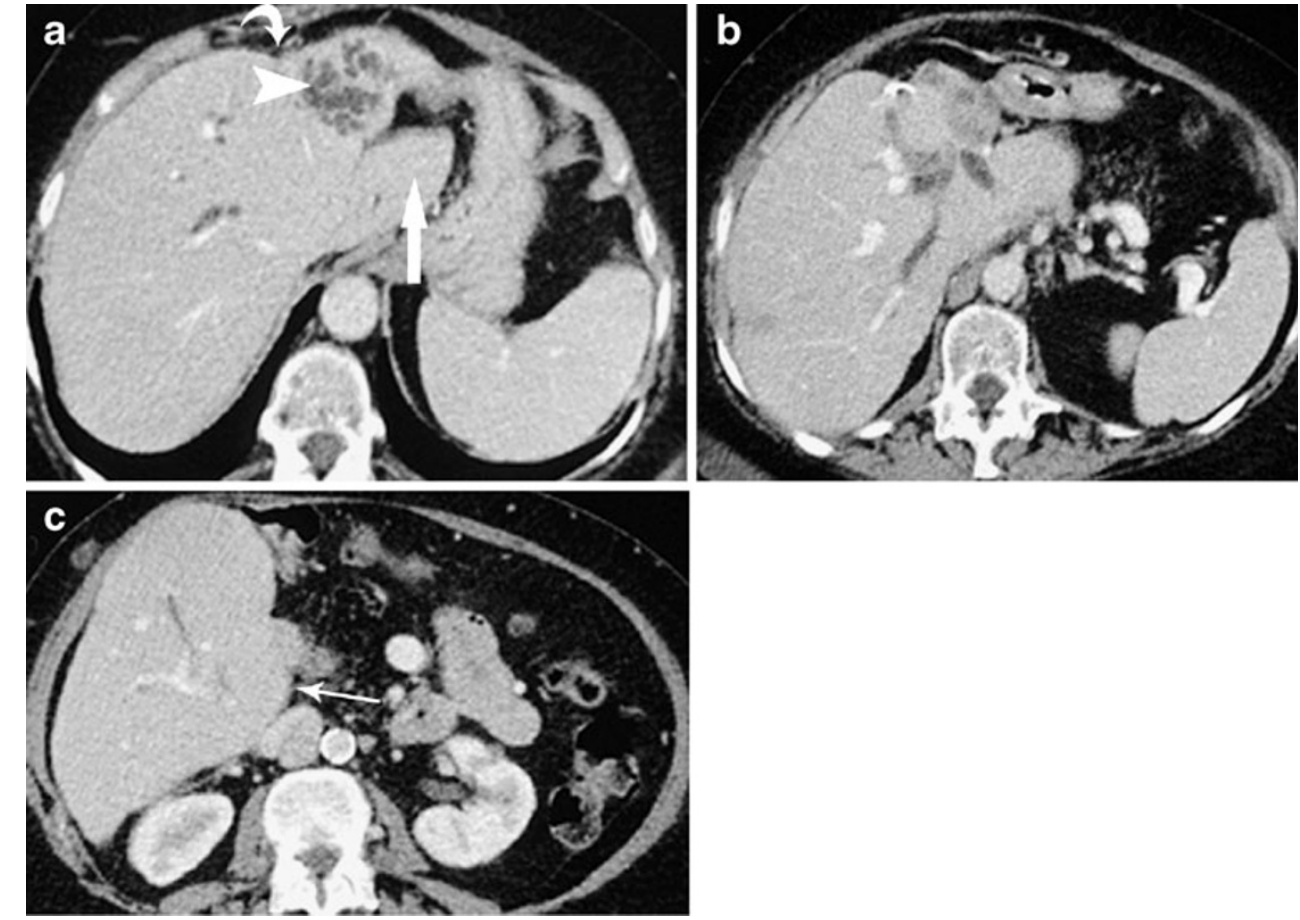
diagnosis of atrophy and is a useful sign when determining the lobar or segmental distribution of atrophy.

\section{CT attenuation and MRI signal intensity}

Atrophied liver parenchyma relative to non-atrophied parenchyma may show lower attenuation on pre-contrast CT [16], higher attenuation during the hepatic arterial phase of contrast-enhanced CT [4, 17], decreased signal intensity on T1-weighted MRI and increased signal intensity on T2weighted MRI $[17,18]$.

Some of these features are illustrated in Fig. 5, where the atrophied parenchyma shows lower attenuation on pre-
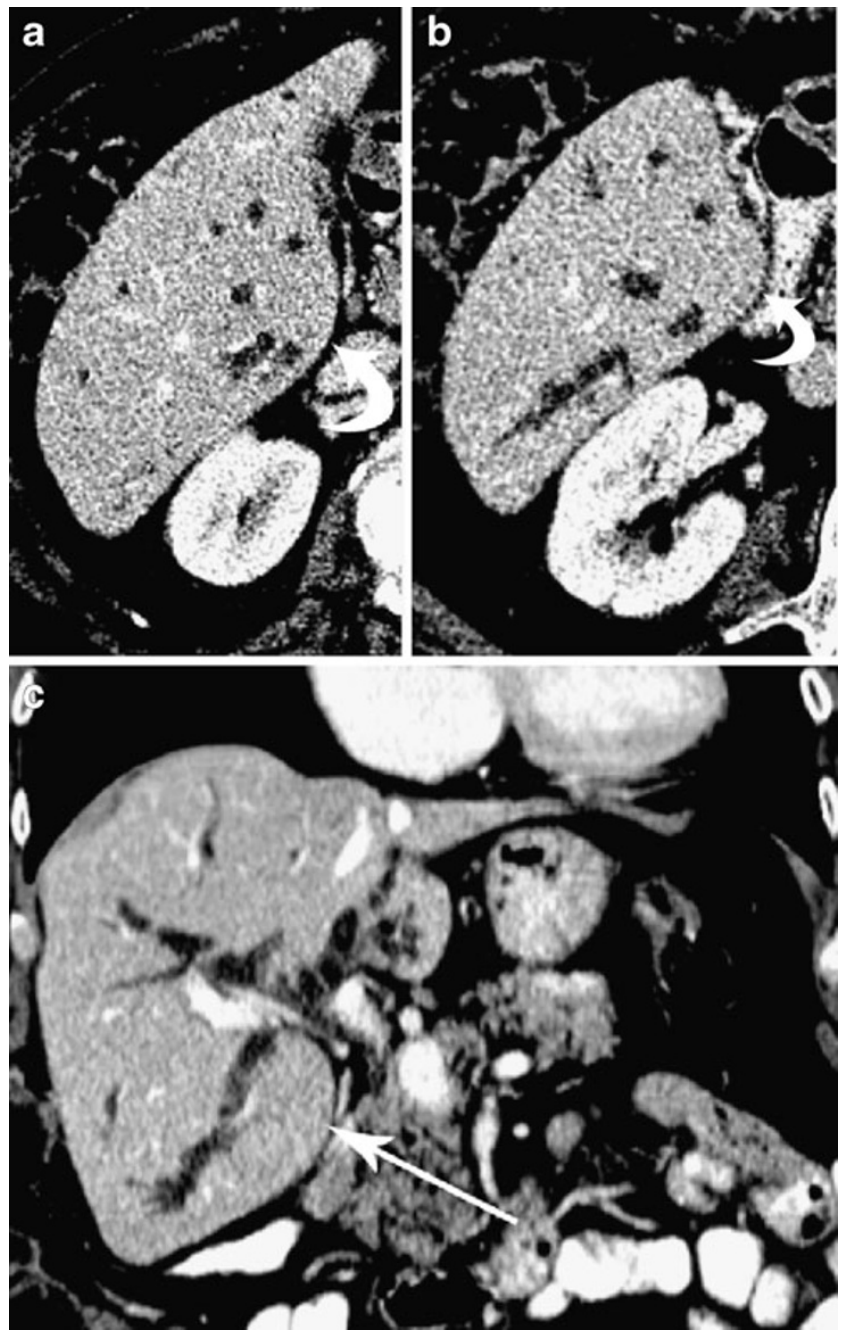

Fig. 4 a-c A patient with atrophy of the left lobe and hypertrophy of the right lobe associated with cholangiocarcinoma. a-c Portal venous phase contrast-enhanced CT. a, b A small portion of the atrophied left lobe is visible (a); the visceral surface of the hypertrophied right lobe appears "bulbous" (curved arrows). c The increase in dimensions and inferior extent of the hypertrophied right lobe is more apparent in the coronal plane. The bulbous visceral surface of the hypertrophied right lobe is again demonstrated (arrow) contrast $\mathrm{CT}$, and lower signal intensity on $\mathrm{T} 1$-weighted MRI.

An explanation for these findings on pre-contrast $\mathrm{CT}$ and T1-weighted MRI could be the increased water content in that portion of liver resulting from oedema, arterio-portal shunting [19] and/or fibrosis [17] within the atrophied lobe/ segment.

In our experience, these $\mathrm{CT}$ attenuation and MRI signal intensity changes are not present in the majority of patients with lobar or segmental liver atrophy.

The atrophic liver parenchyma may also show lower attenuation on $\mathrm{CT}$ intravenous cholangiography (CT-IVC)
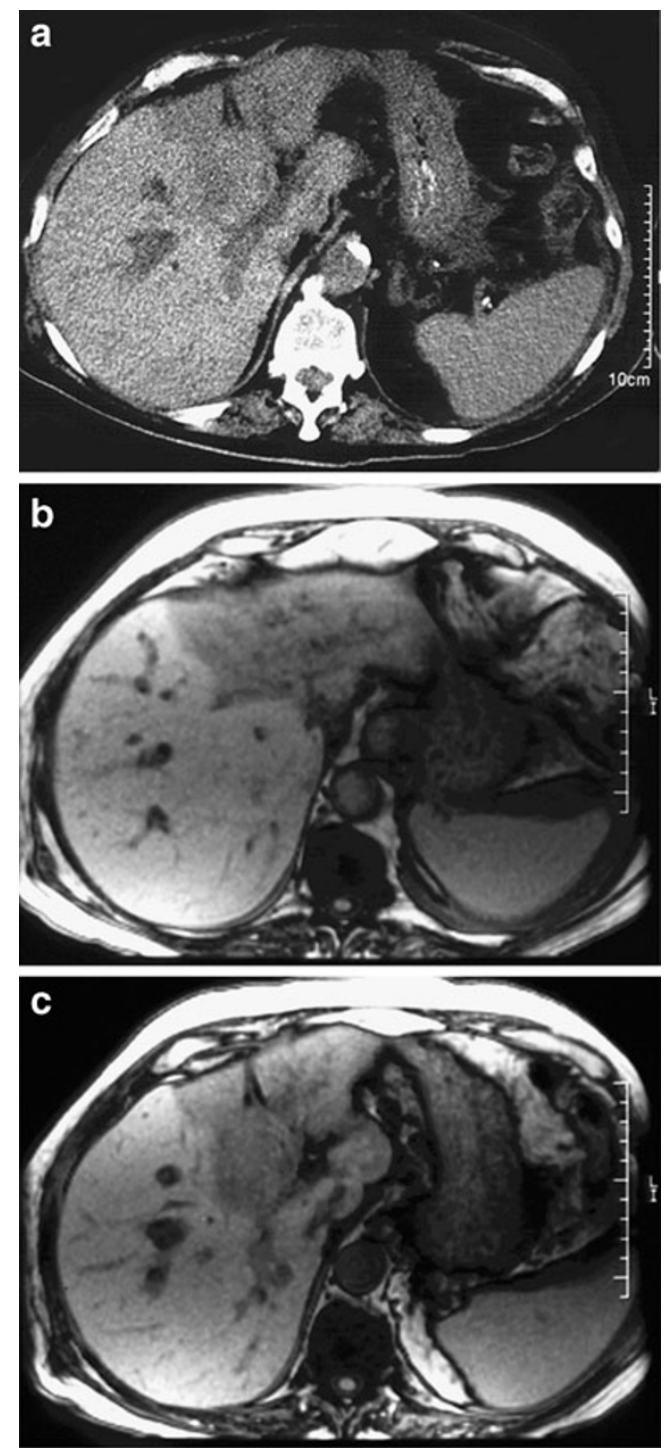

Fig. 5 a-c A patient with left lobe atrophy associated with malignant hilar biliary obstruction due to metastasis of colorectal origin. The left portal vein branch is occluded. a Non-contrast-enhanced CT. The atrophied lobe shows lower attenuation than the non-atrophied lobe. b, c T1-weighted MRI. The atrophied left lobe shows lower signal intensity than the hypertrophied right lobe 
(Fig. 2). This finding is explained by the reduced hepatocyte function and/or number, and consequently decreased contrast medium uptake and excretion by the atrophic parenchyma.

\section{Compensatory hypertrophy}

Lobar volume increase and duct spreading

In patients with lobar or segmental liver atrophy, the non-atrophied parenchyma may undergo compensatory hyperplasia (more often referred to as hypertrophy, even though histologically the volume increase results from hyperplasia) (Figs. 1, 2, 3, 4, 6, 7, 8) [3, 5]. Compensatory hypertrophy is common [4, 8] and is important to recognise as it helps with recognition of contralateral atrophy, and has critical importance in planning surgical resection or interventional approaches to biliary decompression $[3,7]$.

Contrary to the crowded appearance of bile ducts in an atrophic lobe, those in a hypertrophied lobe appear relatively spread out (Figs. 1,2). This spreading is most obvious when it affects the left lateral segments with resulting increased separation of the segment II and III ducts (Fig. 2c).

The relative occurrence of compensatory hypertrophy in patients with right versus left lobe atrophy has received little attention in the English literature. In two small series, one reported an equal rate of compensatory hypertrophy [8], whereas the other showed a higher rate in patients with right lobe atrophy [20].

In our experience compensatory hypertrophy is more common in patients with right lobe atrophy than left lobe atrophy, possibly because right lobe hypertrophy is more difficult to perceive.

In left lobe hypertrophy, the main increase in dimensions occurs in the axial plane on $\mathrm{CT}$, which is the standard imaging plane (Figs. 1, 2). Normally, as mentioned above, the left liver lobe is substantially smaller than the right. Therefore, left lobe hypertrophy as a percentage change from baseline volume may be more substantial and therefore more obvious. Left lobe hypertrophy extends towards the left (Fig. 2a), and the left lateral segments tend to extend inferiorly into the central abdomen (Fig. 1c).

In cases of right lobe hypertrophy, the increase in dimensions is more obvious in the coronal plane (Fig. 4c). In the axial plane the hypertrophied right lobe (or segments) tends to show a prominent convex or "bulbous" contour of the visceral surface (Figs. 3, 4, 7, 8). To our knowledge, this finding has not previously been described. As the right lobe is normally larger than the left the degree of hypertrophy has to be more marked for it to be conspicuous. The recognition of the "bulbous" contour sign as well as the use of coronal CT or MRI should allow more ready recognition of right lobe hypertrophy.

\section{Caudate lobe}

In the presence of right or left lobe atrophy the caudate lobe may be atrophied or hypertrophied or neither (Figs. 1, 2, 3). In our experience the latter is the most common.

The development of atrophy or hypertrophy is likely to be determined by the involvement of caudate bile duct obstruction, which is variable in hilar malignant obstruction, as well as the status of the left portal vein, which provides the dominant portal supply to the caudate lobe [20,21].

\section{Segmental atrophy/hypertrophy associated with hilar biliary anatomical variation}

Unilateral biliary obstruction tends to result in a pattern of lobar atrophy with contralateral lobar hypertrophy [6]. In at least some cases of lobar atrophy associated with cholangiocarcinoma, the tumour arises more peripherally, at
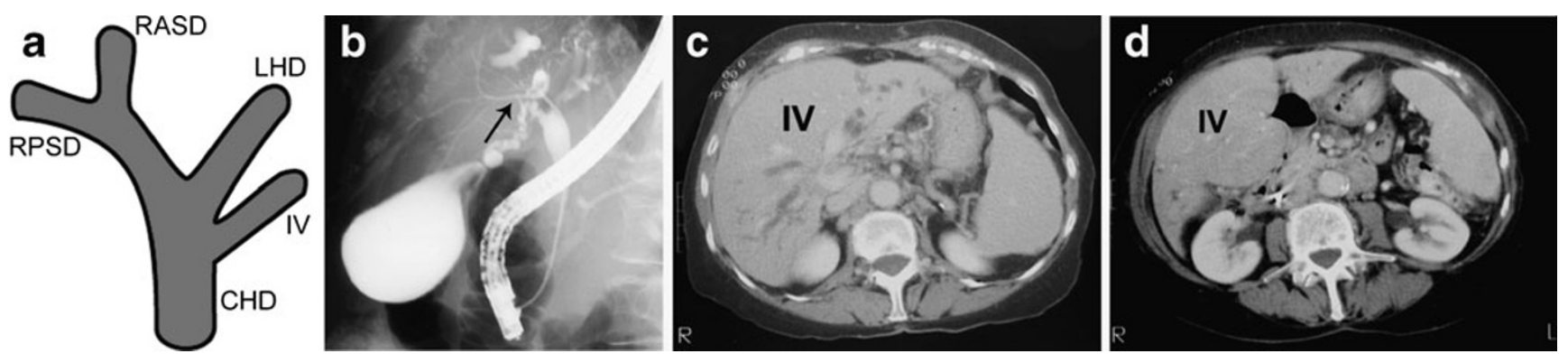

Fig. 6 a-d A patient with right lobe and left lateral segmental atrophy associated with cholangiocarcinoma. a Diagram representing a variant segment IV duct draining into the common hepatic duct $(C H D)$ (RASD right anterior sectoral duct, RPSD right posterior sectoral duct, $R H D$ right hepatic duct, $L H D$ left hepatic duct, $I V$ segment four duct). b Endoscopic retrograde cholangiopancreatogram (ERCP) demonstrates the normal calibre segment IV duct (arrow) draining into the common hepatic duct, inferior to the hilar stricture. c, d CT demonstrates atrophy of the right lobe and left lateral segments, and hypertrophy of segment IV 

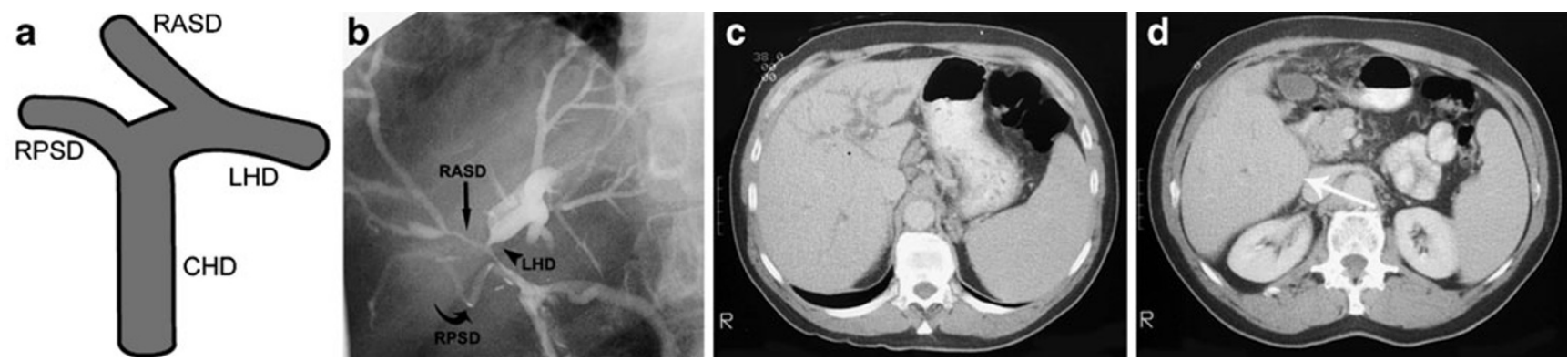

Fig. 7 a-d A patient with left lobe and right anterior segmental atrophy associated with cholangiocarcinoma. a Diagram representing a variant right anterior sectoral duct (RASD) draining into the left hepatic duct (LHD) (RPSD right posterior sectoral duct, CHD common hepatic duct). b Percutaneous transjejunal cholangiogram demonstrates a variant RASD (straight arrow) draining into the LHD (arrowhead). The RPSD (curved arrow) is seen. c, $\mathbf{d}$ CT demonstrates atrophy of the left lobe and right anterior segments, and hypertrophy of the right posterior segments. Note the convex ("bulbous") visceral surface of the hypertrophied right posterior segments inferiorly (curved arrows)

whether it is via an endoscopic or percutaneous approach. Stenting an atrophic lobe can increase the risk of cholangitis developing subsequently [7], is unlikely to relieve jaundice and does not reverse the process of atrophy [3].

Three patterns of segmental atrophy/hypertrophy associated with hilar biliary anatomical variations are illustrated in Figs. 6, 7, 8, all in patients with cholangiocarcinoma.

\section{Conclusion}

Recognising the presence of lobar or segmental atrophy in patients with biliary obstruction is important because of the implications for treatment. The imaging signs are a combination of morphological changes resulting from volume loss and increase respectively in atrophy and hypertrophy, as well as attenuation changes on CT and CT-IVC, and signal changes on MRI. Hilar biliary anatomical variants result in a range of unusual but predictable patterns of segmental atrophy with or without hypertrophy.
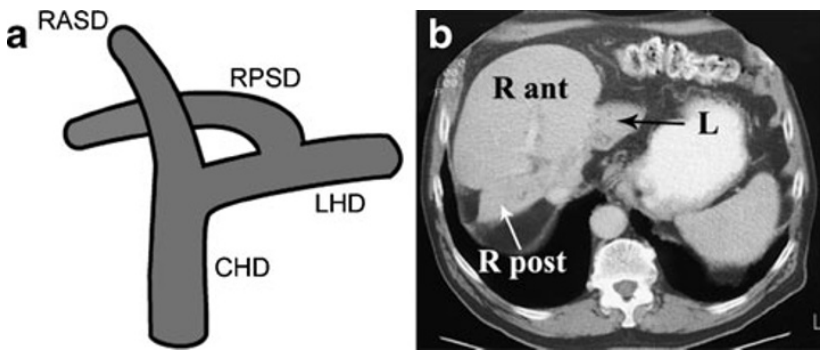

Fig. 8 a-d A patient with segmental atrophy associated with cholangiocarcinoma; the right posterior sectoral duct drained into the left hepatic duct. a Diagram representing a variant right posterior sectoral duct (RPSD) draining into the left hepatic duct ( $L H D)$ (RASD right anterior sectoral duct, $C H D$ common hepatic duct). b CT demonstrates atrophy of the left lobe (black arrow) and right posterior
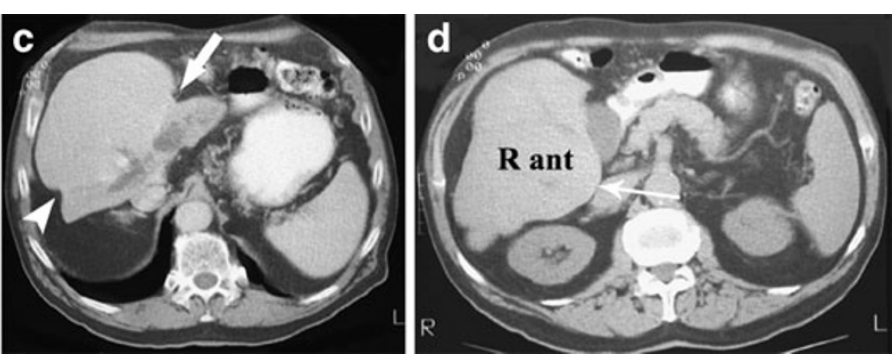

segments (white arrow), and hypertrophy of the right anterior segments. c Note the "step" in the contour between the left lobe/right anterior segments (arrow), and between the right anterior segments/ right posterior segments (arrowhead). d Note the "bulbous" contour of the visceral surface of the hypertrophied right anterior segments inferiorly (arrow) 


\section{References}

1. Czerniak A, Soreide O, Gibson RN et al (1986) Liver atrophy complicating benign bile duct strictures. Surgical and interventional radiologic approaches. Am J Surg 152:294-300

2. Ham JM (1990) Lobar and segmental atrophy of the liver. World J Surg 14:457-462

3. Fausto N, Hadjis NS, Fong Y (2000) Liver hyperplasia, hypertrophy and atrophy, and the molecular basis of liver regeneration. In: Blumgart LH, Fong Y (eds) Surgery of the liver and biliary tract, 3rd edn. Saunders, New York, pp 65-83

4. Takayasu K, Muramatsu Y, Shima Y et al (1986) Hepatic lobar atrophy following obstruction of the ipsilateral portal vein from hilar cholangiocarcinoma. Radiology 160:389-393

5. Hadjis NS, Adam A, Gibson RN et al (1989) Nonoperative approach to hilar cancer determined by the atrophy-hypertrophy complex. Am J Surg 157:395-399

6. Schalm L, Bax H, Mansen B (1956) Atrophy of the liver after occlusion of the bile ducts or portal vein and compensatory hypertrophy of the un-occluded portion and its clinical importance. Gastroenterology 31(2):131-155

7. Gibson RN (1994) Percutaneous transhepatic cholangiography and biliary drainage. In: Adam A, Gibson RN (eds) Practical interventional radiology of the hepatobiliary system and gastrointestinal tract. Arnold, London, pp 14-31

8. Hadjis NS, Hemingway A, Carr D et al (1986) Liver lobe disparity consequent upon atrophy. Diagnostic, operative and therapeutic considerations. J Hepatol 3:285-293

9. Hann LE, Getrajdman GI, Brown KT et al (1996) Hepatic lobar atrophy: association with ipsilateral portal vein obstruction. AJR Am J Roentgenol 167:1017-1021

10. Myracle MR, Stadalnik RC, Blaisdell FW et al (1981) Segmental biliary obstruction: diagnostic significance of bile duct crowding. AJR Am J Roentgenol 137(1):169-71

11. Humar A, Ramcharan T, Sielaff TD et al (2001) Split liver transplantation for two recipients: an initial experience. Am J Transplant 1:366-372. doi:10.1002/lt.2001
12. Kawasaki S, Makuuchi M, Matsunami H et al (1993) Preoperative measurement of segmental liver volume of donors for living related liver transplantation. Hepatology 18:1115-1120

13. Hermoye L, Laamari-Azjal I, Cao Z et al (2005) Liver segmentation in living liver transplant donors: comparison of semiautomatic and manual methods. Radiology 234:171-178. doi:10.1148/radiol.2341031801

14. Kamel IR, Kruskal JB, Warmbrand G et al (2001) Accuracy of volumetric measurements after virtual right hepatectomy in potential donors undergoing living adult liver transplantation. Am J Roentgenol 176:483-487

15. Chaib E, Morales MM, Bordalo MB et al (1995) Predicting the donor liver lobe weight from body weight for split-liver transplantation. Braz J Med Biol Res 28:759-760

16. Demaerel P, Marchal G, Van Steenbergen W et al (1989) CT demonstration of right hepatic lobe atrophy. J Comput Assist Tomogr 13:351-353

17. Yamashita Y, Takahashi M, Kanazawa S et al (1992) Parenchymal changes of the liver in cholangiocarcinoma: CT evaluation. Gastrointest Radiol 17:161-166

18. Siegelman ES, Outwater EK, Piccoli CW et al (1997) MRI of benign and malignant hepatic lobar atrophy. Clin Imaging 21(1):43-50

19. Itai Y, Ohtomo K, Kokubo T et al (1988) Segmental intensity differences in the liver on MR images: a sign of intrahepatic portal flow stoppage. Radiology 167:17-19

20. Ishida H, Naganuma K, Konno T et al (1998) Lobar atrophy of the liver. Abdom Imaging 23:150-153. doi:10.1007/s002619900309

21. Ortale JR, Borges Keiralla LC (2004) Anatomy of the portal branches and the hepatic veins in the caudate lobe of the liver. Surg Radiol Anat 26:384-391. doi:10.1007/s00276-004-0240-4

22. Smadja C, Blumgart LH (1994) The biliary tract and the anatomy of biliary exposure. In: Blumgart LH (ed) Surgery of the liver and biliary tract. Churchill Livingstone, Philadelphia

23. Couinaud C (1957) Le foie. Etudes anatomiques et chirurgicales, vol. 1. Masson, Paris.

24. Hadjis NS, Carr D, Hatzis G et al (1987) Anicteric presentation of hilar cholangiocarcinoma. Anatomical and pathological considerations. Dig Surg 4:119-122 